\title{
BIN ZHANG
}

\section{Revêtements étales abéliens de courbes génériques et ordinarité}

\author{
Annales de la faculté des sciences de Toulouse $\sigma^{e}$ série, tome $1, \mathrm{n}^{\mathrm{O}} 1$ \\ (1992), p. 133-138 \\ <http://www.numdam.org/item?id=AFST_1992_6_1_1_133_0>
}

(C) Université Paul Sabatier, 1992, tous droits réservés.

L'accès aux archives de la revue «Annales de la faculté des sciences de Toulouse » (http://picard.ups-tlse.fr/ annales/) implique l'accord avec les conditions générales d'utilisation (http://www.numdam.org/conditions). Toute utilisation commerciale ou impression systématique est constitutive d'une infraction pénale. Toute copie ou impression de ce fichier doit contenir la présente mention de copyright.

\section{Numdam}

Article numérisé dans le cadre du programme Numérisation de documents anciens mathématiques http://www.numdam.org/ 


\title{
Revêtements étales abéliens de courbes génériques et ordinarité
}

\author{
BIN ZHANG ${ }^{(1)}$
}

\begin{abstract}
RÉSUMÉ. - Soit $L$ un corps algébriquement clos de caractéristique $p>0$. Considérons une $L$-courbe générique de genre $g$. On montre par dégénérescence que tout revêtement étale abélien de la courbe générique de groupe d'ordre premier à $p$ est ordinaire (théorème 3.1 ). In résulte alors de [3] que tout revêtement étale abélien de la courbe générique est ordinaire.
\end{abstract}

Abstract. - We study the ordinarity of étale abelian coverings of the generic curve of given genus $g$ in positive characteristic $p$. We prove that all étale abelian coverings of the generic curve are ordinary.

\section{Préliminaire}

Soient $C$ une courbe sur un corps séparablement clos $k$ et $c$ un point fermé de $C$. Rappelons que $c$ est un point double ordinaire si le complété $\left(\mathcal{O}_{C, c}\right)^{\wedge}$ de l'anneau local $\mathcal{O}_{C, c}$ est isomorphe au quotient $k \llbracket x, y \rrbracket /(x y)$ de l'anneau des séries formelles $k \llbracket x, y \rrbracket$ en deux variables. En particulier $c$ est un point rationnel.

Soient $S$ un schéma et $g$ un entier positif. Rappelons qu'une courbe semistable (resp. stable) de genre $g$ au-dessus de $S$ est un morphisme propre et plat $f: \mathcal{C} \rightarrow S$ dont les fibres géométriques sont des schémas $\mathcal{C}_{\bar{s}}$ réduits, connexes, de dimension 1 et satisfaisant aux deux (resp. trois) conditions suivantes :

i) $\mathcal{C}_{\bar{s}}$ n'admet que des points doubles ordinaires pour singularités;

ii) $\operatorname{dim} H^{1}\left(\mathcal{C}_{\bar{s}}, \mathcal{O}_{\mathcal{C}_{\bar{s}}}\right)=g$.

(1) Université Paul Sabatier, U.F.R. Mathématiques, 118 route de Narbonne, 31062 Toulouse Cedex (France) 


\section{Bin Zhang}

(resp. iii) si $E$ est une composante rationnelle de $\mathcal{C}_{\bar{s}}$, alors $E$ coupe les autres composantes irréductibles en au moins trois points.)

Dans toute la suite, on fixe un entier $g \geq 2$. Soit $k$ un corps séparablement clos de caractéristique $p>0$. D'après [2], on a une courbe stable universelle $Z_{g} \rightarrow H_{g}$ où $H_{g}$ est un $k$-sous-schéma d'un schéma de Hilbert convenable avec les propriétés suivantes (cf. [2]).

\section{PropriÉtÉS}

- Toute courbe stable sur $k$ de genre $g$ est isomorphe à une fibre de $Z_{g} \rightarrow H_{g}$.

- $H_{g}$ est géométriquement irréductible et lisse sur $k$, et l'ensemble des $x \in H_{g}$ tels que la fibre de $Z_{g}$ soit lisse est un ouvert dense de $H_{g}$.

Considérons une clôture algébrique $L$ de $k(\eta)$, où $\eta$ est le point générique de $H_{g}$. On obtient ainsi une courbe générique de genre $g: X=$ $Z_{g} \times{ }_{H_{g}}$ Spec $L$. Cette courbe générique $X / L$ est propre, lisse et connexe. On va étudier l'ordinarité des revêtements étales abéliens de $X$.

Soit $S$ un schéma de caractéristique $p>0$. Soit $f: X \rightarrow S$ un morphisme de schémas. Soit $X^{(p)}=X \times{ }_{S} S$ où le morphisme $S \rightarrow S$ est le morphisme de Frobenius absolu. On note $F: X \rightarrow X^{(p)}$ le $S$-morphisme de Frobenius relatif.

DÉfinition 1.1. - Soit $X$ une courbe semi-stable sur un corps $K$ de caractéristique $p>0$. Le morphisme de Frobenius relatif $F: X \rightarrow X^{(p)}$ induit une application $k$-linéaire de $F^{*}: H^{1}\left(X^{(p)}, \mathcal{O}_{X(p)}\right) \rightarrow H^{1}\left(X, \mathcal{O}_{X}\right)$. On dit que $X$ est ordinaire si $F^{*}$ est bijectif.

\section{Remarques 1.2}

i) Les courbes elliptiques ordinaires sur un corps de caractérisque $p>0$ sont celles d'invariant de Hasse non nul. Les courbes elliptiques non ordinaires correspondent à un nombre fini de valeurs de l'invariant $j$.

ii) Soit $X$ une courbe semi-stable sur un corps $K$ de caractéristique $p>0$. Soit $K^{\prime}$ une extension de $K$. On note $X^{\prime}$ la courbe $X \bigotimes_{K} K^{\prime}$. Alors $X$ est ordinaire si et seulement si $X^{\prime}$ est ordinaire.

iii) Soit $S$ un schéma intègre noethérien de caractéristique $p>0$ et $f: \mathcal{C} \rightarrow S$ une courbe semi-stable. Notons ord $(\mathcal{C} / S)$ l'ensemble des $s \in S$ tels que $\mathcal{C}_{s}$ soient ordinaires. Alors ord $(\mathcal{C} / S)$ est un ouvert de $S$. 
iv) Soient $K$ un corps algébriquement clos de caractéristique $p>0, E$ et $E^{\prime}$ deux courbes elliptiques sur $K$. Si $f: E^{\prime} \rightarrow E$ est une isogénie, alors $E$ est ordinaire si et seulement si $E^{\prime}$ est ordinaire.

2. Genre et normalisation d'une courbe semi-stable

Proposition 2.1. - Soient $K$ un corps séparablement clos et $C$ une courbe sur $K$ semi-stable de genre $g$. On note $C^{\prime}$ le normalisé de $C$, $m$ le nombre de points singuliers de $C$, et $n$ le nombre des composantes irréductibles de $C$. Alors $g+n-m-1$ est la somme des genres des normalisés des composantes irréductibles de $C$.

Notons que le normalisé $C^{\prime}$ est lisse sur $K$. Soient $C=\bigcup_{1 \leq i \leq n} C_{i}$ où $C_{i}$ sont les composantes irréductibles de $C, f: C^{\prime} \rightarrow C$ le morphisme canonique et $C_{i}^{\prime}$ le normalisé de $C_{i}$. Les $C_{i}^{\prime}$ sont les composantes de $C^{\prime}$. Nous avons une suite exacte induite par le morphisme canonique injectif $h: \mathcal{O}_{C} \rightarrow f_{*} \mathcal{O}_{C^{\prime}}$ :

$$
0 \rightarrow \mathcal{O}_{C} \rightarrow f_{*} \mathcal{O}_{C^{\prime}} \rightarrow Q \rightarrow 0
$$

où $Q=f_{*} \mathcal{O}_{C^{\prime}} / \mathcal{O}_{C}$.

$Q$ est concentré aux points singuliers de $C$ et chaque point singulier de $C$ se relève en deux points distincts de $C^{\prime}$ car $C$ est semi-stable, et $Q$ est de longueur 1 en chaque point double de $C$.

$$
\begin{aligned}
0 & \rightarrow H^{0}\left(C, \mathcal{O}_{C}\right) \rightarrow H^{0}\left(C, f_{*} \mathcal{O}_{C^{\prime}}\right) \rightarrow H^{0}(C, Q) \rightarrow \\
\rightarrow H^{1}\left(C, \mathcal{O}_{C}\right) & \rightarrow H^{1}\left(C, f_{*} \mathcal{O}_{C^{\prime}}\right) \rightarrow H^{1}(C, Q)=0
\end{aligned}
$$

D'où une suite exacte

$$
0 \rightarrow K \rightarrow \prod_{1 \leq i \leq n} K \rightarrow \prod_{1 \leq i \leq m} K \rightarrow H^{1}\left(C, \mathcal{O}_{C}\right) \rightarrow H^{1}\left(C^{\prime}, f_{*} \mathcal{O}_{C^{\prime}}\right) \rightarrow 0
$$

et on en déduit

$$
\sum_{1 \leq i \leq n}\left(\text { genre de } C_{i}^{\prime}\right)=g+n-m-1 .
$$


Corollaire 2.2. - Soient $K$ un corps séparablement clos et $C$ une courbe semi-stable de genre $g$. Soit $C^{\prime}$ sa normalisée. Alors le " genre de $C^{\prime}$ " est $\leq g$. Supposons de plus que les composantes $C_{i}$ de $C$ soient lisses sur $K$ et forment une "chaîne ", c'est-à-dire $n=m+1$, et $C$ a au plus deux points singuliers sur chaque $C_{i}$. Alors

$$
\sum_{1 \leq i \leq n}\left(\text { genre de } C_{i}\right)=g
$$

COROLlaIRE 2.3. - Une courbe semi-stable sur un corps séparablement clos de caractéristique $p>0$ est ordinaire si et seulement si sa normalisée est "ordinaire", c'est-à-dire les composantes de sa normalisée sont ordinaires.

\section{Revêtements étales abéliens de la courbe générique}

Retournons à la situation du paragraphe 1 . Nous pouvons maintenant énoncer le résultat principal que nous avons en vue.

ThÉoRÈme 3.1. - Soit $X / L$ une courbe générique de genre $g \geq 2$. Soit $Y$ un revêtement étale connexe galoisien de $X$ de groupe $G$ commutatif d'ordre premier à $p$. Alors $Y$ est ordinaire.

Remarque 3.2. - En particulier $X$ est ordinaire, ce qui est classique (cf. $[5])$.

Démonstration du théorème 3.1.- Prenons $g$ courbes elliptiques ordinaires sur $k$. Il existe une courbe stable $C_{0}$ de genre $g$ sur $k$ qui est une " chaîne " constituée de ces $g$ courbes elliptiques ordinaires.

$C_{0}$ correspond alors à une fibre de $Z_{g} / H_{g}$ en un point rationnel $x$ de $H_{g}$.

Il existe un anneau de valuation discrète $R$ tel que, si on note $\xi$ le point générique de $S=\operatorname{Spec} R$ et $s$ le point fermé de $S$, on ait un morphisme $f: S \rightarrow H_{g}$ qui induit un isomorphisme $K(R) \cong k(\eta)$ et $f(s)=x, f(\xi)=\eta$ ([4], II (7.1.9)), où $K(R)$ est le corps des fractions de $R$.

Soit $\mathcal{X}=Z_{g} \times_{H_{g}} \operatorname{Spec} R$. On obtient une courbe stable $\mathcal{X} / S$, la fibre générique géométrique est $X / L$, la fibre spéciale est une courbe stable $C=C_{0} \bigotimes_{k} k(s)$ qui est une " chaîne " constituée de $g$ courbes elliptiques ordinaires. 
On introduit quelques notations :

$E:=$ le corps des fractions de $R$;

$\mathcal{J}:=\operatorname{Pic}^{0}(\mathcal{X} / S), J_{L}:=\operatorname{Pic}^{0}(X / L), J_{E}:=\operatorname{Pic}^{0}\left(\left(\mathcal{X} \bigotimes_{R} E\right) / E\right)$ (cf. [1], chap. 8 et 9$)$;

$G:=G_{S}$ le $S$-schéma en groupes constant associé à $G, G_{E}:=G \bigotimes_{R} E$;

$G^{\prime}:=$ le dual de Cartier de $G\left(=\underline{\operatorname{Hom}}_{S-G r}\left(G, \mathbb{G}_{m, S}\right)\right)$;

$G_{E}^{\prime}:=G^{\prime} \otimes_{R} E, G_{L}^{\prime}:=G^{\prime} \bigotimes_{R} L$.

Lemme 3.3. - $\mathcal{J}$ est un schéma abélien.

D'après [1] section $9.3, \mathcal{J}$ est un $S$-schéma en groupe lisse. La fibre spéciale de $\mathcal{J}$ est un schéma abélien d'après le choix de $C$ et [1] exemple 9.2.8, et par suite $\mathcal{J}$ est un schéma abélien.

Comme $L$ est algébriquement clos, on peut factoriser $\operatorname{Spec} L \rightarrow \operatorname{Spec} R$ à travers un hensélisé strict de $R$, donc supposer $R$ strictement hensélien. Alors d'après [7], (6.2), nous avons un diagramme commutatif :

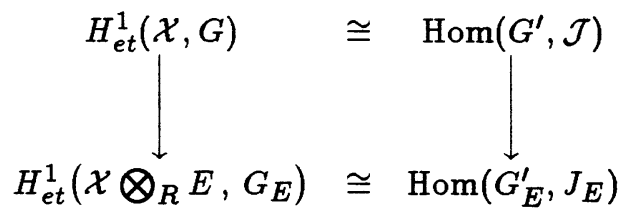

Le revêtement $Y / X$ correspond à un morphisme injectif $h: G_{L}^{\prime} \rightarrow J_{L}$. Soit $n=\sharp(G)$. $h$ correspond à un morphisme injectif $h^{\prime}: G_{L}^{\prime} \rightarrow\left(J_{L}\right)_{n}=$ $\mathcal{J}_{n} \otimes_{R} L$ (indice $n$ désigne le noyau de la multiplication par $n$ ). $n$ est premier à $p$, donc $G^{\prime}$ et $\mathcal{J}_{n}$ sont des $S$-groupes finis étales. $R$ est strictement hensélien, donc $G^{\prime}$ et $\mathcal{J}_{n}$ sont $R$-isomorphes à des réunions disjointes de Spec $R$, donc $h^{\prime}$ provient d'un morphisme $r: G_{E}^{\prime} \rightarrow\left(J_{E}\right)_{n}$ et $r$ se prolonge en un morphisme $G^{\prime} \rightarrow \mathcal{J}_{n}$ qui correspond à un morphisme $G^{\prime} \rightarrow \mathcal{J}$ prolongeant $G_{E}^{\prime} \rightarrow J_{E}$.

Il résulte alors de $(*)$ que le revêtement étale $Y / X$ se prolonge en un revêtement étale $\mathcal{Y} / \mathcal{X}$ connexe galoisien de groupe de Galois $G$.

Le revêtement $\mathcal{Y} / \mathcal{X}$ donne sur la fibre fermée un revêtement étale galoisien $q: D \rightarrow C$ de groupe $G$.

Considérons le morphisme induit $q^{\prime}: D^{\prime} \rightarrow C^{\prime}$ où $D^{\prime}$ et $C^{\prime}$ sont les normalisés respectifs de $D$ et $C$. On a $D^{\prime}=D \times{ }_{C} C^{\prime}$ car le morphisme $q$ est étale. 
$C^{\prime}$ est réunion disjointe de courbes elliptiques ordinaires par construction de $C$, donc $D^{\prime}$ est ordinaire (remarque 1.2.iv), et par suite $D$ est ordinaire (corollaire 2.3). Donc $Y$ est ordinaire (remarque 1.2.iii).

Le théorème et [3], corollaire 1.8.3, entraînent le résultat suivant.

CorollaIRE 3.4. - Tout revêtement étale abélien connexe d'une courbe générique est ordinaire.

\section{Références}

[1] Bosch (S.), Lutkebohmert (W.) et Raynaud (M.) . - Néron Models, A Series of modern Surveys in Mathematics, Springer-Verlag, 21 (1990).

[2] Deligne (P.) et Mumford (D.) . - The irreducibility of the space of curves of given genus,

Publ. Math. IHES, 36 (1969) pp. 75-110.

[3] CREW (R.M.) - - Etale p-covers in caracteristic p, Compositio Math., 52 (1984) pp. 31-45.

[4] Grothendieck (A.) et Dieudonné (J.) . - Éléments de géométrie algébrique, Publ. Math. IHES, 8.

[5] Kовцiтz (N.) . - p-adic variation of the zeta- function over familles of varieties defined over finite field,

Compositio Math., $31 \mathrm{n}^{\circ} 2$ (1975) pp. 119-218.

[6] Mumford (D.) . - Abelian varieties,

Oxford Univ. Press, Oxford (1970).

[7] Raynaud (M.) . - Spécialisation du foncteur de Picard, Publ. Math. IHES, 38 (1970) pp. 27-76. 